\title{
Estrategia de evaluación formativa en educación superior: el caso del módulo de investigación educativa
}

\author{
Formative evaluation strategy in higher education: the case of the \\ educational research module
}

\section{* Carlos Miranda Carvajal \& ** Pablo Castillo Armijo}

Miranda, C., \& Castillo, P. (2020). Estrategia de evaluación formativa en educación superior: el caso del módulo de investigación educativa. Revista Convergencia Educativa, 8, diciembre, 31-44. DOI: http://doi.org/10.29035/rce.8.31

[Recibido: 16 junio, 2020 / Aceptado: 09 diciembre, 2020]

\section{RESUMEN}

Se desarrolló la aplicación de dos estrategias simultaneas de evaluación en el curso de investigación educacional, buscando comprobar si existen diferencias significativas en los resultados cuantitativos que expresan, a nivel de formación inicial docente, el desarrollo de las competencias asociadas a la asignatura. Las estrategias de evaluación formativa compuestas de retroalimentación y el uso de rúbricas de trabajo, orientó un desarrollo de informes semanales en los que se trabajó específicamente el contenido tratado más el uso de bibliografía especializada; esta estrategia de evaluación se consideró como parte del proceso de aprendizaje como plantea la teoría, llegando a la aplicación de una prueba objetiva de alternativas simples al final del proceso lectivo, la que se intervino con dos estrategias del modelo Master \& Learning, para comprobar si los resultados salían de la curva normal. Se comprobó que los resultados obtenidos por los estudiantes se elevan por medio de la aplicación de evaluación formativa, reflejando un desarrollo de las competencias disciplinares de la asignatura; de la misma manera se comprobó que la aplicación de una prueba objetiva genera resultados que reflejan la curva normal en donde se concentran los alcances cuantitativos en los puntajes medios.

Palabras clave: evaluación del aprendizaje, investigación educacional, evaluación formativa, evaluación objetiva, formación inicial docente.

\footnotetext{
* Pontificia Universidad Católica de Valparaíso, Quilpué, Chile. ORCID ID: https://orcid.org/0000-0003-0570-3496

** Universidad Católica del Maule, Talca, Chile. ORCID ID: https://orcid.org/0000-0002-5708-4618
} 
Miranda, C., \& Castillo, P. (2020). Estrategia de evaluación formativa en educación superior: el caso del módulo de investigación educativa. Revista Convergencia Educativa 8, diciembre, 31-44. DOI: http://doi.org/10.29035/rce.8.31

\section{ABSTRACT}

The application of two simultaneous evaluation strategies was developed in the course of educational research. The aim was to recognise the existence of significant differences in the quantitative results that express, at the level of initial teacher training, the development of the competences associated with the subject. The formative evaluation strategies composed of feedback and the use of working rubrics guided the development of weekly reports in which the content was specifically worked on in addition to the use of specialised literature. This evaluation strategy was considered as part of the learning process as stated in the theory, reaching the application of an objective test of simple alternatives at the end of the teaching process, which was intervened with two strategies of the master \& learning model to check whether the results deviated from the normal curve. It was verified that the results obtained by the students are raised using the application of formative evaluation, reflecting the development of the disciplinary competences of the subject, in the same way, it was verified that the application of an objective test, generates results that reflect the normal curve where the quantitative reaches are concentrated in the average scores.

Key words: Learning Evaluation, Educational Research, Formative Evaluation, Objective Evaluation, Initial Teacher Training.

\section{Introducción}

Los procesos de formación docente en Chile, específicamente en instituciones de educación superior estatales, están supeditados a la búsqueda del desarrollo de competencias que hacen que los educadores y educadoras se conviertan en figuras integrales, quienes conocen los procesos, posibles productos y desarrollos de los procesos, en los que se indican las constantes actualización, innovación e investigación.

A nivel general, en el sistema educativo chileno de formación inicial docente, se propone como un módulo importante la Investigación Educacional. En el caso específico revisado de una universidad pública y especializada en pedagogía, la competencia a desarrollar por los estudiantes es el "analizar problemas educativos en contextos diversos, desde la investigación educacional”. La medición de la competencia se realiza de manera cuantitativa a nivel de escala 1.0 a 7.0, como forma estandariza, donde la nota 4.0 es la mínima para aprobar. En este último punto es donde centramos nuestra atención, ya que creemos que deben existir otras formas de comprobar el aprendizaje de tan compleja competencia y que se hará fundamental para el posterior desarrollo de trabajos y/o tesis de grado conducentes a la Licenciatura en Educación.

De esta manera se busca evidenciar los alcances que ha tenido la implementación de un trabajo que concibe a la evaluación de procesos, auténtica y contextualizada, como una oportunidad de aprendizaje para todos los estudiantes y, de esta manera, lograr la apropiación de conocimientos y conceptos claves y la vinculación de los estudiantes con escenarios y procesos naturales de la investigación y las coincidencias y/o diferencias de esta propuesta con un modelo de evaluación objetivo psicométrico que sitúa a la evaluación al final del proceso de aprendizaje, poniendo en contrapunto dos modelos de evaluación y 
Miranda, C., \& Castillo, P. (2020). Estrategia de evaluación formativa en educación superior: el caso del módulo de investigación educativa. Revista Convergencia Educativa 8, diciembre, 31-44. DOI: http://doi.org/10.29035/rce.8.31

buscando responder a la interrogante: ¿existen diferencias en las calificaciones logradas en cada uno de los modelos de evaluación empleados en el curso?

\section{Marco teórico}

La necesidad de transitar desde una representación clásica de incorporación de la evaluación al aprendizaje en contextos académicos hacia una que efectivamente se centre en los aprendizajes de los propios sujetos, su contexto, capacidades e intereses. En esta última perspectiva el proceso de evaluación involucra, en una etapa inicial, la participación del profesor, quien promueve y orienta su desarrollo en el estudiante, y para ello debe reconocer, primeramente, la importancia que tiene el propósito de la evaluación y la técnica evaluativa a usar en el desarrollo del proceso, y especialmente, la importancia para potenciar los aprendizajes (Lara \& Larrondo, 2008).

La creación de una cultura evaluativa, tal como lo aborda Núñez (2001), apunta a fomentar una participación voluntaria y honesta de todos los actores involucrados en el proceso educativo, lo cual repercutirá en un mejoramiento en las prácticas de cada uno de los participantes de la evaluación, principalmente utilizando la retroalimentación como el principal foco del proceso.

El objetivo de una nueva cultura evaluativa será, por tanto, un cambio cultural del ámbito pedagógico, donde se promueva el desarrollo de prácticas de mayor autonomía y confianza en el autoaprendizaje de los estudiantes, en reemplazo de una cultura evaluativa basada en el control y la sanción que prevalece en el sistema educativo y los procesos de evaluación (Santos, 1988, 1990, 1993, 1999, 2013, Santos \& Szmolka, 2015, Ahumada, 2005, Anijovich, 2013, Bolívar, 1995, Perrenoud, 2004, Stake, 2006).

Con el pasar de los años los objetivos que buscan los procesos evaluativos han ido cambiando y, por ende, las estrategias que se utilizan para ello también lo han hecho, guiados principalmente por los resultados meta analíticos (Bloom, 1984) que proponen estrategias de enseñanza que involucran a la evaluación como pilar de los procesos educativos, ya que la evaluación como sanción, puede ser entendida como aquella evaluación que tiende a actuar para muchos estudiantes como un sistema que registra el fracaso, y que lo hace público (Jackson, 1991). Este planteamiento evaluativo tradicional, parte de la consideración del error como motivo de sanción, de tal modo que la evaluación es sinónimo automático de calificación (López, 2005).

El desarrollo de una evaluación alternativa orientada hacia el proceso, consiste en delinear, obtener, procesar y proveer información válida, confiable y oportuna sobre el mérito del aprendizaje de los estudiantes, con el fin de emitir un juicio de valor que permita tomar diversos tipos de decisiones. El rol del evaluador, será por tanto, generar procesos de diálogos, comprobación y retroalimentación (Santos, 1988, 1990, 1993, 1999, 2013, Santos \& Szmolka, 2015; Ahumada, 2005, Anijovich, 2013, Bolívar, 1995, Perrenoud, 2004, Stake, 2006), definiendo que "la evaluación es una parte de los procesos de enseñanza y 
Miranda, C., \& Castillo, P. (2020). Estrategia de evaluación formativa en educación superior: el caso del módulo de investigación educativa. Revista Convergencia Educativa 8, diciembre, 31-44. DOI: http://doi.org/10.29035/rce.8.31

aprendizaje y no un apéndice, un complemento o un adorno" (Santos, 1993, p. 173) tomando en cuenta estas consideraciones, es la evaluación autentica (Condemarín \& Medina, 2000), que propone el utilizar diferentes estrategias y procedimientos evaluativos, que en su espectro más amplio, debiera incluir situaciones de la vida real y problemas significativos de naturaleza compleja.

Ahumada (2001) propone tomar en cuenta las raíces de las concepciones de aprendizajes que se encuentran en las prácticas reflexivas (Schön, 1992, Novak \& Gowin, 1983), aplicadas a los estudiantes, y la consideración de la evaluación como retroalimentación, lo que permitiría fomentar entre ellos su responsabilidad en el aprendizaje, a partir de la identificación de sus fortalezas y debilidades, con el fin de incrementar su autoconfianza y capacidad de logro.

Prieto y Contreras, 2008:6), se suman a este nuevo proceso de evaluación auténtica, destacando el valor formativo que puede tener en los estudiantes, haciéndolos capaces de llegar a procesos metacognitivos superiores.

Se enfatiza la función formativa de la evaluación y se le significa como una práctica tendiente, tanto a conocer el nivel de comprensión de los estudiantes acerca de los conocimientos y nivel de desarrollo de habilidades y destrezas propias de un determinado contenido enseñado, como a reflexionar y explicitar los fundamentos de las prácticas implementadas por los profesores.

El confiar en los estudiantes, dotarlos del poder de evaluar sus aciertos y errores, abre la posibilidad de entablar una nueva relación de colaboración y cambio de los roles tradicionales de los principales actores del acto educativo, por una parte, los profesores dejarán atrás el estigma del sancionador del error y por otra parte, los estudiantes le darán sentido al conocimiento adquirido, no solo por el hecho de una calificación, sino por el deseo de mejorar cualitativamente sus actuaciones y reflexiones.

\section{Metodología}

Se utilizo un diseño cuantitativo experimental de carácter transaccional (Briones, 1992, Bunge, 1997, Hernández, Fernández \& Baptista, 2010). Los participantes fueron estudiantes de cuarto año de formación inicial docente de las carreras de pedagogía de una universidad pública regional y especializada en pedagogía de Chile, siendo un $\mathrm{N}=23$.

Se usaron los modelos evaluación auténtica y evaluación psicométrica, con los instrumentos rúbrica y test objetivo respectivamente, para generar la experiencia de mejora e innovación docente.

\section{Experiencia de innovación educativa}

La experiencia se desarrolló en primera instancia con la aplicación de modelos de evaluación formativa (Glass, 2016) a nivel de trabajos prácticos en dos etapas, con técnicas de evaluación de informe y 
Miranda, C., \& Castillo, P. (2020). Estrategia de evaluación formativa en educación superior: el caso del módulo de investigación educativa. Revista Convergencia Educativa 8, diciembre, 31-44. DOI: http://doi.org/10.29035/rce.8.31

autoevaluación de informe, entregando a los estudiantes una rúbrica de desarrollo. Además de la aplicación de un test objetivo por medio de técnicas pertenecientes al método Master \& Learning (Bloom, 1968) en la etapa final del desarrollo del curso.

Cronograma de actividades académicas del curso (ver Tabla 1):

Tabla 1

Sistematización de actividades en clases.

Clase Momentos de aprendizaje

1 Entrega del programa del curso. Creación de cronograma de entrega de informes y evaluación objetiva, entrega de tabla de especificaciones de la evaluación objetiva.

Entrega de rúbrica para desarrollar los informes y revisión del apartado sobre $1^{\circ}$ informe.

2 Recepción de $1^{\circ}$ informe: "Planteamiento del Problema de Investigación". Revisión de rúbrica en el apartado sobre $2^{\mathrm{O}}$ informe.

3 Recepción del 2o informe: "Marco Teórico". Revisión de rúbrica en el apartado sobre $3^{\circ}$ informe.
Actividades orientadas al proceso de evaluación.

Desarrollo de clase sobre temática de primer informe: "Planteamiento del problema de investigación".

Desarrollo de clase sobre temática de $2^{\circ}$ informe: "Marco Teórico".
Retroalimentación sobre evaluación del $1^{\underline{0}}$ informe "Planteamiento del Problema" por grupo de trabajo, devolución de informe y calificación del trabajo. Desarrollo de clase sobre temática de 3 o informe: "Marco Metodológico".

4 Recepción de $3^{\text {o }}$ informe: "Marco Metodológico". Desarrollo de clase sobre revisión bibliográfica sobre metodología de la investigación.

Retroalimentación sobre evaluación del $2^{2}$ informe "Marco Teórico" por grupo de trabajo, devolución de informe y calificación del trabajo.

Retroalimentación sobre evaluación del 3o informe "Marco Metodológico" por grupo de trabajo, devolución de informe y calificación del trabajo. Problema de Investigación", con correcciones de las observaciones de la primera entrega.

Desarrollo de clase sobre trabajo de campo y recolección de datos.

6 Recepción segunda entrega de $2^{\text {}}$ informe: "Marco Teórico", con correcciones de las observaciones de la primera entrega. Desarrollo de clase sobre trabajo de campo y recolección de datos.

(

Retroalimentación sobre evaluación de la segunda entrega de $1^{\circ}$ informe "Planteamiento del Problema" por grupo de trabajo, devolución de informe y calificación del trabajo por medio de la autoevaluación.

7 Recepción segunda entrega de 3o informe: "Marco Metodológico", con correcciones de las observaciones de la primera entrega. Desarrollo de clase sobre análisis de datos cualitativos.

Retroalimentación sobre evaluación de la segunda entrega de 2o informe "Marco Teórico" por grupo de trabajo, devolución de informe y calificación del trabajo por medio de la autoevaluación.

8 Desarrollo de clase sobre análisis de datos cuantitativos.
Retroalimentación sobre evaluación de la segunda entrega de $3^{\circ}$ informe "Marco Metodológico" por grupo de trabajo, devolución de informe y calificación del trabajo por medio de la autoevaluación.

Aplicación de Evaluación Objetiva en tres etapas: Desarrollo de test individual en 30 minutos.

Desarrollo de test en parejas 20 minutos.

Desarrollo de test en grupos de trabajo con uso de textos y apuntes en 15 minutos.

Fuente: elaboración propia. 


\section{Clase Momentos de aprendizaje}

10 Desarrollo de foro interno sobre fortalezas y debilidades en el desarrollo del proyecto, poniendo puntos comparativos en el desarrollo de las diferentes etapas.

\section{Actividades orientadas al proceso de evaluación.}

Entrega de proyecto de investigación por grupo, el proyecto se compone de las tres etapas de entrega formativa: Planteamiento del problema, Marco teórico, Marco metodológico.

Fuente: elaboración propia.

La revisión de la rúbrica de trabajo se realizó clase a clase entes de las entregas de cada informe, según los rubros y la asignación de puntajes detallada (ver Tabla 2).

Tabla 2

Especificaciones de la rúbrica de desarrollo de informes y proyecto de investigación.

\begin{tabular}{lcccc}
\hline $\begin{array}{l}\text { Etapa de } \\
\text { la investigación }\end{array}$ & $\begin{array}{c}\text { Nivel de } \\
\text { desarrollo bajo }\end{array}$ & $\begin{array}{c}\text { Nivel de } \\
\text { desarrollo medio bajo }\end{array}$ & $\begin{array}{c}\text { Nivel de } \\
\text { desarrollo medio alto }\end{array}$ & $\begin{array}{c}\text { Nivel de } \\
\text { desarrollo alto }\end{array}$ \\
\hline $\begin{array}{l}\text { Planteamiento del } \\
\text { problema }\end{array}$ & $(1,0-3,9)$ & $(4,0-4,9)$ & $(5,0-5,9)$ & $(6,0-7,0)$ \\
Marco teórico & $(1,0-3,9)$ & $(4,0-4,9)$ & $(5,0-5,9)$ & $(6,0-7,0)$ \\
\hline Marco metodológico & $(1,0-3,9)$ & $(4,0-4,9)$ & $(5,0-5,9)$ & $(6,0-7,0)$ \\
\hline
\end{tabular}

Fuente: elaboración propia.

Los avances a nivel de calificación en el proceso de entrega, revisión y retroalimentación se aprecian de la siguiente forma (ver Tabla 3). 
Tabla 3

Calificaciones por estudiantes en entregas formativas y entrega fina de informes.

\begin{tabular}{|c|c|c|c|c|c|c|c|}
\hline Estudiante & $E n 1 P P$ & $E n 1 M T$ & En $1 M M$ & $E n 2 P P$ & En $2 M T$ & $E n 2 M M$ & $X E v$ \\
\hline 1 & 20 & 10 & 10 & 49 & 64 & 64 & 36 \\
\hline 2 & 20 & 10 & 10 & 49 & 64 & 64 & 36 \\
\hline 3 & 20 & 10 & 10 & 49 & 64 & 64 & 36 \\
\hline 4 & 47 & 49 & 61 & 63 & 64 & 64 & 58 \\
\hline 5 & 47 & 49 & 61 & 63 & 64 & 64 & 58 \\
\hline 6 & 47 & 49 & 61 & 63 & 64 & 64 & 58 \\
\hline 7 & 47 & 49 & 61 & 63 & 64 & 64 & 58 \\
\hline 8 & 47 & 49 & 61 & 63 & 64 & 64 & 58 \\
\hline 9 & 40 & 10 & 22 & 20 & 66 & 66 & 37 \\
\hline 10 & 40 & 10 & 22 & 20 & 66 & 66 & 37 \\
\hline 11 & 40 & 10 & 22 & 20 & 66 & 66 & 37 \\
\hline 12 & 40 & 10 & 22 & 20 & 66 & 66 & 37 \\
\hline 13 & 40 & 10 & 22 & 20 & 66 & 66 & 37 \\
\hline 14 & 40 & 10 & 22 & 20 & 66 & 66 & 37 \\
\hline 15 & 56 & 58 & 54 & 66 & 57 & 57 & 58 \\
\hline 16 & 56 & 58 & 54 & 66 & 57 & 57 & 58 \\
\hline 17 & 56 & 58 & 54 & 66 & 57 & 57 & 58 \\
\hline 18 & 58 & 50 & 59 & 64 & 65 & 65 & 60 \\
\hline 19 & 58 & 50 & 59 & 64 & 65 & 65 & 60 \\
\hline 20 & 58 & 50 & 59 & 64 & 65 & 65 & 60 \\
\hline 21 & 58 & 50 & 59 & 64 & 65 & 65 & 60 \\
\hline 22 & 56 & 58 & 54 & 66 & 57 & 57 & 58 \\
\hline 23 & 10 & 50 & 59 & 64 & 65 & 65 & 52 \\
\hline
\end{tabular}

En: entrega; PP: planteamiento del problema; MT: marco teórico; MM: marco metodológico; X Ev: nota evaluación. Fuente: elaboración propia.

Los alcances a nivel de evaluación objetiva, proponen la aplicación de un test de tipo objetivo compuesto de 28 ítems planteados de manera simpática según la taxonomía de Bloom que considera como contenido la bibliografía obligatoria del curso, apuntes de clase y desarrollo de trabajo práctico sobre técnicas cualitativas y cuantitativas de investigación lo que se observa en las especificaciones entregadas a los estudiantes (ver Tabla 4). 
Tabla 4

Especificaciones test objetivo.

\begin{tabular}{|c|c|c|c|c|c|c|}
\hline & $\begin{array}{c}\text { Conocimiento } \\
\text { (1 punto })\end{array}$ & $\begin{array}{c}\text { Comprensión } \\
\text { (3 puntos) }\end{array}$ & $\begin{array}{l}\text { Aplicación } \\
\text { (5 puntos) }\end{array}$ & $\begin{array}{c}\text { Síntesis } \\
\text { (7 puntos) }\end{array}$ & $\begin{array}{c}\text { Análisis } \\
\text { (9 puntos) }\end{array}$ & Total \\
\hline Texto 1 & $\begin{array}{l}1 \text { ítem } \\
1 \text { punto }\end{array}$ & $\begin{array}{c}1 \text { ítem } \\
3 \text { puntos }\end{array}$ & -- & -- & $\begin{array}{c}1 \text { ítem } \\
9 \text { puntos }\end{array}$ & $\begin{array}{c}3 \text { ítems } \\
13 \text { puntos }\end{array}$ \\
\hline Texto 2 & $\begin{array}{l}2 \text { ítems } \\
2 \text { puntos }\end{array}$ & $\begin{array}{l}2 \text { ítems } \\
6 \text { puntos }\end{array}$ & & & $\begin{array}{c}1 \text { ítem } \\
9 \text { puntos }\end{array}$ & $\begin{array}{c}5 \text { ítems } \\
17 \text { puntos }\end{array}$ \\
\hline Texto 3 & ------- & $\begin{array}{c}4 \text { ítems } \\
12 \text { puntos }\end{array}$ & ---- & --- & ------- & $\begin{array}{c}4 \text { ítems } \\
12 \text { puntos }\end{array}$ \\
\hline Texto 4 & $\begin{array}{l}1 \text { ítem } \\
1 \text { punto }\end{array}$ & $\begin{array}{c}1 \text { ítem } \\
3 \text { puntos }\end{array}$ & ---- & --- & $\begin{array}{c}2 \text { ítems } \\
18 \text { puntos }\end{array}$ & $\begin{array}{c}4 \text { ítems } \\
22 \text { puntos } \\
\end{array}$ \\
\hline Texto 5 & ------- & ------- & ------- & ------ & $\begin{array}{c}5 \text { ítems } \\
45 \text { puntos }\end{array}$ & $\begin{array}{c}5 \text { ítems } \\
45 \text { puntos }\end{array}$ \\
\hline $\begin{array}{c}\text { Téc. } \\
\text { Cualitativa }\end{array}$ & $\begin{array}{l}2 \text { ítems } \\
2 \text { puntos }\end{array}$ & $\begin{array}{l}2 \text { ítems } \\
6 \text { puntos }\end{array}$ & $--\cdot$ & $\begin{array}{c}2 \text { ítems14 } \\
\text { puntos }\end{array}$ & ------- & $\begin{array}{c}6 \text { ítems } \\
22 \text { puntos }\end{array}$ \\
\hline $\begin{array}{c}\text { Téc. } \\
\text { Cuantitativa }\end{array}$ & ------- & -- & $\begin{array}{c}5 \text { ítems } \\
25 \text { puntos }\end{array}$ & ------- & - & $\begin{array}{c}5 \text { ítems } \\
25 \text { puntos }\end{array}$ \\
\hline TOTAL & $\begin{array}{l}5 \text { ítems } \\
6 \text { puntos }\end{array}$ & $\begin{array}{l}10 \text { ítems } \\
30 \text { puntos }\end{array}$ & $\begin{array}{c}5 \text { ítems } \\
25 \text { puntos }\end{array}$ & $\begin{array}{c}2 \text { ítems } \\
14 \text { puntos }\end{array}$ & $\begin{array}{c}6 \text { ítems } \\
81 \text { puntos }\end{array}$ & $\begin{array}{c}28 \text { ítems } \\
156 \text { puntos }\end{array}$ \\
\hline
\end{tabular}

Fuente: elaboración propia.

La aplicación del instrumento se desarrolló de la siguiente manera, apelando a una adaptación del modelo Master \& Learning:

1aㅡ Etapa, 30 minutos: respuesta directa. Los estudiantes responden de forma individual el test con un tiempo de un minuto por respuesta, se apunta ala respuestas de 16 ítems de las habilidades básicas como correctos, teniendo una ponderación de $50 \%$ del test respondido en la primera etapa.

2a Etapa, 20 minutos: tutoría en la respuesta. Los estudiantes responden en parejas los restantes ítems del test, apelando a la tutoría entre pares para el desarrollo de los ítems de habilidades superiores, es importante que también se considera como estrategias el intercambio de respuestas a nivel de habilidades bajas y su revisión por parte de los estudiantes.

3a Etapa, 15 minutos: tutoría en la respuesta + paginas paralelas de información. En esta etapa se busca la utilización de información de carácter bibliográfico, dando la instrucción de agruparse entre 4 y 5 estudiantes y hacer uso de apuntes, fotocopias, libros y trabajos desarrollados, esto con el fin de potenciar el acercamiento a la respuesta correcta que pueden tener los estudiantes, por medio de la reinstrucción generada entre pares con bases bibliográficas a favor de las respuestas correctas. 
Los resultados obtenidos en esta evaluación fueron (ver Tabla 5).

Tabla 5

Notas test objetivo.

\begin{tabular}{|c|c|}
\hline Estudiante & NOTA PRUEBA \\
\hline 1 & 10 \\
\hline 2 & 10 \\
\hline 3 & 10 \\
\hline 4 & 22 \\
\hline 5 & 22 \\
\hline 6 & 30 \\
\hline 7 & 33 \\
\hline 8 & 33 \\
\hline 9 & 33 \\
\hline 10 & 34 \\
\hline 11 & 34 \\
\hline 12 & 34 \\
\hline 13 & 35 \\
\hline 14 & 39 \\
\hline 15 & 39 \\
\hline 16 & 39 \\
\hline 17 & 40 \\
\hline 18 & 41 \\
\hline 19 & 41 \\
\hline 20 & 41 \\
\hline 21 & 42 \\
\hline 22 & 47 \\
\hline 23 & 52 \\
\hline
\end{tabular}

Fuente: elaboración propia.

Para un total de 23 estudiantes, se logra la aprobación del 50\%, donde las notas mínimas observadas corresponden a la no presentación de los estudiantes a la evaluación, teniendo en cuenta la no contemplación de estas notas mínimas, y la inexistencia de notas máximas, la evaluación y su planteamiento como tipo objetivo cumple con su rol de agrupación de resultados en la zona media de la curva, es decir entre 2,2 y 5,3 lo que corresponde completamente a la teoría psicométrica de evaluación estandarizada de 
contenidos (Adkins, 1971, Bloom, Hastings \& Madaus, 1975, Pizarro, 1985, 2004, 2008, 2010, Allen, 2000, Stake, 2006, Santos, 2014).

La comparación de los alcances a nivel de calificación en los dos procesos evaluativos, notas finales del proceso y nota en test objetivo, se observan gráficamente de la siguiente manera (ver Figuras 1 y 2 ).

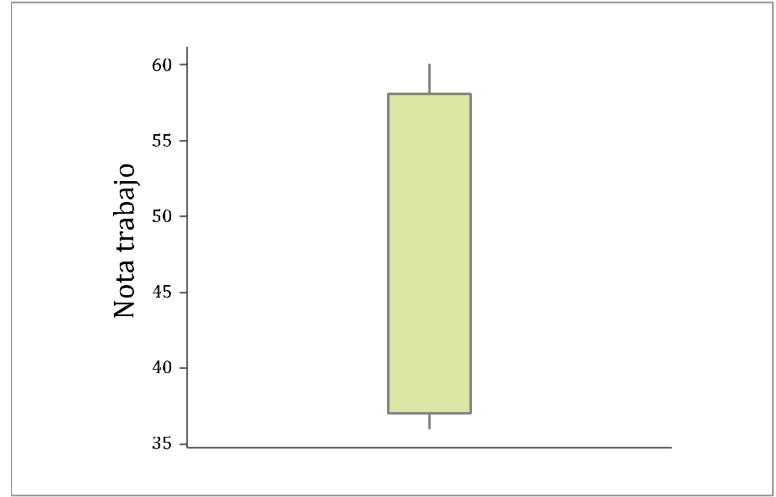

Figura 1. Notas finales trabajo. Fuente: elaboración propia.

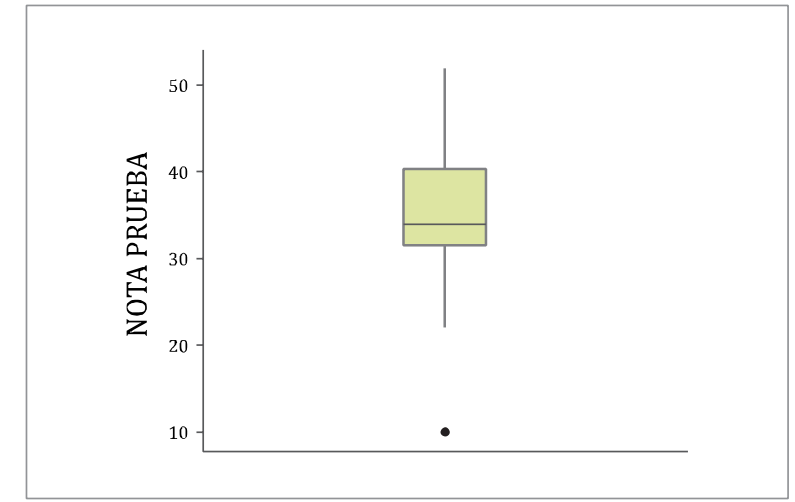

Figura 2. Notas test objetivo. Fuente: elaboración propia.

Los gráficos muestran que los alcances difieren, aun obedeciendo a la misma asignatura, idéntica muestra y tiempos lectivos, lo que se explica en la naturaleza de los procesos de evaluación empleados. Por su parte en el proceso práctico se observa una mayor dispersión de los resultados, con notas extremas y una media elevada a nivel curso. Por su parte el proceso objetivo, presenta una agrupación mayor de los participantes, y una media menor, ubicada bajo la línea de aprobación, con muy pocos alcances elevados y pocos casos descendidos.

\section{Conclusiones}

Las conclusiones de la experiencia se detallan en un orden de logro de los objetivos planteados para el desarrollo de la asignatura:

a) Sobre el uso de Evaluación Formativa (Bloom, 1984, Glass, 2016) y Retroalimentación: a nivel de logro, se demuestra en los procesos prácticos de construcción de informes de investigación entre las semanas 2 y 8, reflejan un mayor avance en la apropiación y aplicación de los aprendizajes, donde las evidencias manifiestan que la mantención constante en procesos de investigación educativa, hace que se genere lo que plantean Perines \& Campaña (2019), como la generación de un habitus investigativo, lo que es equivalente a la propuesta de generación de habitus reflexivo a nivel aula (Schön, 1992, Novak \& Gowin, 1983, Perrenoud, 2004).

b) Al fijarnos en los resultados y como éstos pueden reflejar los aprendizajes de los estudiantes, encontramos que existen coincidencias entre los resultados y la propuesta revisada, ya que claramente 
la evaluación formativa (Bloom, 1984, Glass, 2016), permite el logro de mayores calificaciones y de mejoras sustantivas entre las diferentes evaluaciones desarrolladas en el proceso, más aun, considerando que la constante retroalimentación desarrolladas hace que el modelo evaluativo adoptado, tome sentido en su orientación de la evaluación como parte del proceso de aprendizaje (Santos, 1988, 1990, 1993, 1999, 2013, Santos \& Szmolka, 2015, Ahumada, 2005, Anijovich, 2013, Bolivar, 1995, Perrenoud, 2004, Stake, 2006).

c) Sobre el uso de evaluación objetiva, se observa que se mantienen las normas estándar de resultados para este tipo de test según la teoría psicométrica (Adkins, 1971, Bloom, Hastings \& Madaus, 1975, Pizarro, 1985, 2004, 2008, 2010, Allen, 2000, Stake, 2006, Santos, 2014), concentrando los resultados al centro de la escala y dejando los extremos bajos y altos de la misma, sin concentración de sujetos.

d) Sobre la adaptación en la aplicación del instrumento, el agregar las etapas de tutoría en la respuesta y páginas paralelas de información (Bloom, 1968), se afirma que estas no poseen influencias en los alcances métricos, por lo que, a nivel psicométrico, no se observan variaciones en las notas académicas obtenidas.

e) Como conclusión general, se considera que el fortalecimiento de las competencias investigativas de los docentes en formación, se ven favorecidas por la práctica investigativa temprana, la que, a nivel de aula, se potencia altamente por un modelo de evaluación formativa (Bloom, 1984, Glass, 2016), cumpliendo con lo planteado a nivel del catálogo de productividad de Glass (2016), que propone a este modelo como el primer modelo potenciador de los aprendizajes con un efecto de tamaño 0,90.

Finalmente, los alcances a nivel de notas académicas del modelo de evaluación psicométrica, proporciona una correcta estandarización de los resultados, lo que no desmerece los indicadores de calidad de la evaluación psicométrica, más bien afirman que la naturaleza de este tipo de evaluación se mantiene al agrupar de manera centralizada los resultados, pero por lo que se puede afirmar que no se reflejan los reales aprendizajes a nivel de notas académicas, observado primero a nivel gráfico y en la poca coincidencia de estos alcances con los obtenidos a nivel del trabajo formativo apoyado de retroalimentación constante. 
Miranda, C., \& Castillo, P. (2020). Estrategia de evaluación formativa en educación superior: el caso del módulo de investigación educativa.

Revista Convergencia Educativa 8, diciembre, 31-44. DOI: http://doi.org/10.29035/rce.8.31

\section{REFERENCIAS BIBLIOGRÁFICAS}

Adkins, D. (1971). Elaboración de test. Ciudad de México: Trillas.

Ahumada, P. (2005). La evaluación auténtica: un sistema para la obtención de evidencias y vivencias de los aprendizajes. Perspectiva educacional, (45), 11-24. Recuperado de https://www.redalyc.org/pdf/3333/333329100002.pdf

Ahumada, P. (2001). La Evaluación en una concepción de Aprendizaje significativo. Valparaíso: Ediciones Universitarias de Valparaíso. Recuperado de http://www.euv.cl/archivos_pdf/evaluacion.pdf

Allen, D. (2000). La evaluación del aprendizaje de los estudiantes. Buenos Aires: Paidós.

Anijovich, R. (2013). La evaluación significativa. Buenos Aires: Paidós.

Bloom, B. (1984). The 2 sigma problem: The search for methods of group instruction as effective as one to one tutoring. Educational Researcher, 13(6), 4-16. DOI: https://doi.org/10.3102/0013189X013006004

Bloom, B. (1968). Learning for Mastery. Instruction and Curriculum. Regional Education Laboratory for the Carolinas and Virginia, Topical Papers and Reprints, Number 1. Evaluation Comment, 1(2), 1-12. Recuperado de https://eric.ed.gov/?id=ED053419

Bloom, B., Hastings, J. \& Madaus, F. (1976). Evaluación de los aprendizajes (tomo I). Ciudad de México: Troquel.

Briones, G. (1992). Métodos y técnicas de investigación para las ciencias sociales. Ciudad de México: Trillas.

Bolívar, A. (1995). La evaluación de valores y actitudes. Madrid: Alauda-Anaya.

Bunge, M. (1997). La Ciencia, su método y su filosofía. Buenos Aires: Sudamericana.

Condemarín, M. \& Medina, A. (2000). Evaluación auténtica de los aprendizajes. Santiago de Chile: Andrés Bello.

Glass, V. G. (2016). One hundred years of research: prudent aspirations. Educational Researcher, 42(2), 6972. DOI: https://doi.org/10.3102/0013189X16639026

Hernández, R., Fernández, C., \& Baptista, P. (2010). Metodología de la Investigación. México D.F.: McGraw Hill.

Jackson, Ph. (1991). La vida en las aulas. Madrid: Morata.

Lara, M., \& Larrondo, T. (2008). La autoevaluación en contextos escolares. Su inclusión en los procesos para el aprendizaje. Pensamiento Educativo, 43(2), 259-270. Recuperado de http://pensamientoeducativo.uc.cl/index.php/pel/article/view/441/904 
Miranda, C., \& Castillo, P. (2020). Estrategia de evaluación formativa en educación superior: el caso del módulo de investigación educativa.

Revista Convergencia Educativa 8, diciembre, 31-44. DOI: http://doi.org/10.29035/rce.8.31

López, V. M. (2005). La evaluación como sinónimo de calificación. Implicaciones y efectos en la Educación y en la Formación del Profesorado. Revista Electrónica Interuniversitaria de Formación del Profesorado, 8(4), 1- 7. Recuperado de https://www.redalyc.org/pdf/2170/217017876007.pdf

Novak, J., \& Gowin, D. (1983). Aprendiendo a Aprender. Barcelona: Martínez-Roca.

Núñez, F. (2001). Así evaluamos. Sinéctica, (19), 89-96. Recuperado de https://www.redalyc.org/articulo.oa?id=99817935009

Perines, H., \& Campaña, K. (2019). La alfabetización de los futuros docentes en investigación educativa: Una reflexión teórica desde el contexto de Chile. Caribeña de Investigación Educativa (RECIE), 3(1), 718. DOI: https://doi.org/10.32541/recie.2019.v3i1.pp7-18

Perrenoud, Ph. (2004). Desarrollar la práctica reflexiva en el oficio de enseñar. Profesionalización y razón pedagógica. Barcelona: Graó.

Pizarro, R. (2010). Mismos SIMCEs, otros análisis II: Comparación y desarrollo. Revista Investigaciones en $\begin{array}{lllll}\text { Educación, } & 10 & \text { (2), } & 105-127 . & \text { Recuperado de }\end{array}$ http://revistas.ufro.cl/ojs/index.php/educacion/article/view/1012

Pizarro, R. (2008). Sistemas educativos formales y efectos Mateo, Regresivo y Robin Hood. Boletín de Investigación Educacional, 23 (2), 13-38. Recuperado de http://www.legibilidadmu.cl/3-.pdf

Pizarro, R. (1985). Teoría del rendimiento académico. Diálogos Educacionales, (6), 30-39.

Prieto, M., \& Contreras, G. (2008). Las concepciones que orientan las practicas evaluativas de los profesores: un problema a develar. Estudios pedagógicos (Valdivia), 34(2), 245-262. DOI: https://dx.doi.org/10.4067/S0718-07052008000200015

Santos Guerra, M. Á. (2014). La evaluación como aprendizaje. Madrid: Narcea.

Santos Guerra, M. Á. (2013). Evaluar es comprender. Buenos Aires: Magisterio del Rio de la Plata.

Santos Guerra, M. Á. (1999). Crítica a la eficacia y eficacia de la crítica. Lo verdadero, lo verosímil y lo verificable en el análisis de las instituciones educativas. En J. F. Angulo Rasco, (Coord.), Escuela pública y sociedad neoliberal (pp. 83-112). España: Miño y Dávila Editores.

Santos Guerra, M. Á. (1993). La evaluación: un proceso de diálogo, comprensión y mejora. Investigación en la Escuela, (20), 23-35. Recuperado de https://idus.us.es/bitstream/handle/11441/59547/La\%20evaluaci\%F3n\%20un\%20proceso\%2 0de\%20di\%E1logo,\%20comprensi\%F3n\%20y\%20mejora.pdf?sequence=1\&isAllowed=y

Santos Guerra, M. Á. (1990). Hacer visible lo cotidiano. Teoría y práctica de la evaluación cualitativa en centros escolares. Madrid: Akal. 
Santos Guerra, M. Á. (1988). La evaluación cualitativa de planes y centros de perfeccionamiento del profesorado: una forma de mejorar la profesionalidad docente. Investigación en la Escuela, (6), 2139. Recuperado de https://idus.us.es/handle/11441/59161?

Santos Guerra, M. Á., \& Szmolka, I. (2015). Reflexionar sobre la evaluación. Experiencias en la Facultad de Políticas y Sociología de la Universidad de Granada. Granada: Universidad de Granada.

Schön, D. (1992). La formación de profesionales reflexivos. Hacia un nuevo diseño de la enseñanza y el aprendizaje en las profesiones. Barcelona: Paidós

Stake, R. (2006). Evaluación comprensiva y evaluación basada en estándares. Barcelona: Graó.

\section{Datos de correspondencia}

\section{Carlos Miranda Carvajal}

Magíster en Evaluación educacional

Pontificia Universidad Católica de Valparaíso

Quilpué, Chile

Dirección postal: 2430000

ORCID: https://orcid.org/0000-0003-0570-3496

Email: carlos.miranda.c1@mail.pucv.cl 